\title{
CLOSED ORBITS OF AN ANOSOV FLOW AND THE FUNDAMENTAL GROUP
}

\author{
TOSHIAKI ADACHI
}

\begin{abstract}
We show that closed orbits of a transitive Anosov flow generate the fundamental group of the base manifold.
\end{abstract}

Introduction. Let $\varphi_{t}: M \rightarrow M$ be a transitive Anosov flow on a compact manifold. Fried [5] showed that $H_{1}(M, Z)$ is generated by homology classes of $\varphi_{t}$-closed orbits. In this note we show that homotopy classes of closed orbits generate the fundamental group $\pi_{1}(M)$. More precisely, for $\gamma \in \pi_{1}(M)$ we denote by $\langle\gamma\rangle$ the conjugacy class in $\pi_{1}(M)$ containing $\gamma$. Each closed orbit $\mathfrak{p}$ defines a conjugacy class $\langle\mathfrak{p}\rangle$. Our main result is

Theorem. If $\varphi_{t}: M \rightarrow M$ is a transitive Anosov flow, then those elements $\gamma \in \pi_{1}(M)$ with $\langle\gamma\rangle=\langle\mathfrak{p}\rangle$ for some $\varphi_{t}$-closed orbit $\mathfrak{p}$ generate $\pi_{1}(M)$.

In some cases each conjugacy class contains an orbit cycle (i.e. a multiple of a closed orbit), hence the theorem trivially holds. But we have many examples of Anosov flows without this property; geodesic flows of surfaces and suspensions of Anosov diffeomorphisms.

We prove the theorem by an elementary argument on graphs. A Markov family for $\varphi_{t}$ defines an oriented graph embedded in $M$ such that each $\varphi_{t}$-closed orbit is approximated by an oriented closed path in this graph. In $\$ 1$ we construct a covering of Markov families with the covering transformation group $\pi_{1}(M)$, and show that the associated graphs are connected. Next we show in $\$ 2$ that the induced homomorphism of the fundamental group of the graph into $\pi_{1}(M)$ is surjective. Since the fundamental group of this graph is generated by free homotopy classes of oriented closed paths, we can get the assertion. In $§ 3$, using our main theorem, we improve Theorem B in [3] which is concerned with the analyticity of $L$-functions associated to $\varphi_{t}$.

The author is grateful to T. Sunada for many discussions with him.

1. A covering of Markov families. Let $\pi: \tilde{M} \rightarrow M$ be the universal covering and $\tilde{\varphi}_{t}: \tilde{M} \rightarrow \tilde{M}$ the covering flow of $\varphi_{t}$. In this section we construct a covering of connected graphs associated to a Markov family for $\varphi_{t}$.

Received by the editors December 31, 1985.

1980 Mathematics Subject Classification (1985 Revision). Primary 58F15; Secondary 58F20.

Key words and phrases. Anosov flow, closed orbit, fundamental group, oriented graph. 
An oriented graph $(V, E)$ consists of a set of vertices $V$ and a set of edges $E \subset V \times V$. We usually identify it with a 1-dimensional $\mathrm{CW}$-complex equipped with an orientation on each 1-simplex. An oriented path $c=\left(v_{0}, \ldots, v_{n}\right),\left(v_{i}, v_{i+1}\right) \in E$, from $v_{0}$ to $v_{n}$ is said to be closed if $v_{0}=v_{n}$. We call $(V, E)$ irreducible if for every pair $(v, w) \in V \times V$ there is an oriented path from $v$ to $w$. An irreducible graph is connected as a CW-complex (the converse is, in general, not true).

We put

$$
C=1+\sup \left\{d\left\langle\tilde{\varphi}_{t}(p), p\right\rangle / t \mid 0<t \leqslant 1, p \in \tilde{M}\right\} .
$$

If $\varepsilon>0$ is small enough, then for each $\tilde{x} \in \tilde{M}$ the $10 \varepsilon C$-neighborhood of $\tilde{x}$ is contained in some covering sheet, and the stable and unstable sets $W_{\varepsilon}^{\mathrm{s}}(x), W_{\varepsilon}^{\mathrm{u}}(x)$ $(x \in M)$ for $\varphi_{t}$ play a role of coordinates (see $\left.[8,9]\right)$.

Let $V$ be a Markov family of size $\alpha$. (Here $\alpha$ is sufficiently small compared with $\varepsilon / 10 C$.) For the definition and the properties of a Markov family, see [4]. We use here the irreducible oriented graph $(V, E)$ defined as follows. Since $V$ is a finite family of disjoint $\varphi_{t}$-local cross sections, for each $x \in \Gamma(V)=\bigcup_{v \in V} v$ one can attach the smallest positive $t(x)(\leqslant \alpha)$ with $\varphi_{t(x)}(x) \in \Gamma(V)$. We define $E$ by

$$
\left\{(v, w) \in V \times V \mid \text { there is } x \in v \cap \Gamma^{\prime}(V) \text { with } T(x) \in w\right\},
$$

where $T: \Gamma(V) \rightarrow \Gamma(V)$ is the bijection given by $T(x)=\varphi_{t(x)}(x)$ and

$$
\Gamma^{\prime}(V)=\left\{x \in \Gamma(V) \mid T^{k}(x) \in \bigcup_{v \in V} \operatorname{Int}(v) \text { for every } k \in \mathbb{Z}\right\}
$$

is a dense subset of $\Gamma(V)$. The transitivity of $\varphi_{t}$ guarantees that $(V, E)$ is irreducible.

We now construct an oriented graph associated to $\tilde{\varphi}_{t}$. Since the diameter of $v \in V$ is smaller than $\alpha$, there exists $\tilde{v} \subset \tilde{M}$ such that

$$
\pi^{-1}(v)=\bigcup_{\gamma \in \pi_{1}(M)} \gamma \tilde{v}, \quad \gamma \tilde{v} \cap \tilde{v}=\varnothing \text { if } \gamma \neq \mathrm{id} .
$$

The family of $\tilde{\varphi}_{t}$-local cross sections $\tilde{V}=\left\{\gamma \tilde{v} \mid v \in V, \gamma \in \pi_{1}(M)\right\}$ similarly yields an oriented graph $(\tilde{V}, \tilde{E})$. Note that the bijection $\tilde{T}: \Gamma(\tilde{V}) \rightarrow \Gamma(\tilde{V})$ satisfies $\pi$ 。 $\tilde{T}=T \circ \pi$ and $\Gamma^{\prime}(\tilde{V})=\pi^{-1}\left(\Gamma^{\prime}(V)\right)$. Therefore, if we define $P: \tilde{V} \rightarrow V$ by $P(\gamma \tilde{v})=v$, then $P:(\tilde{V}, \tilde{E}) \rightarrow(V, E)$ is a covering of oriented graphs with the covering transformation group $\pi_{1}(M)$ (i.e. a covering of CW-complexes with compatible orientations). Although $\tilde{V}$ might be an infinite family, it plays a role of a Markov family for $\tilde{\varphi}_{t}$. One can find a strictly positive function $\tilde{f}$ and a bounded-to-one continuous map $\tilde{\Phi}$ : $\Sigma(\tilde{V}, \tilde{E}, \tilde{f}) \rightarrow \tilde{M}$ such that

(i) $\tilde{\varphi}_{t} \circ \tilde{\Phi}=\tilde{\Phi} \circ \sigma(\tilde{f})_{t}$,

(ii) for each $\tilde{x} \in \Gamma(\tilde{V})$ there is $\xi \in \Sigma(\tilde{V}, \tilde{E})$ with $\tilde{\Phi}(\xi, 0)=\tilde{x}$.

Here $\Sigma(\tilde{V}, \tilde{E}, \tilde{f})$ denotes the suspension with $\tilde{f}$ of the subshift $\Sigma(\tilde{V}, \tilde{E})=\{\xi=$ $\left.\left(\xi^{i}\right) \in \Pi_{\mathbf{Z}} \tilde{V} \mid\left(\xi^{i}, \xi^{i+1}\right) \in \tilde{E}\right\}$ and $\sigma(\tilde{f})_{t}$ the suspension flow.

We devote the rest of this section to showing that $(\tilde{V}, \tilde{E})$ is connected. By the definition of a Markov family we may suppose that for each $\tilde{v} \in \tilde{V}$ there exists a $\tilde{\varphi}_{t}$-local cross section $\tilde{D}$ containing $\tilde{v}$ such that

(1) if $\tilde{v} \cap \tilde{\varphi}_{[-\alpha, \alpha]}\left(\tilde{v}^{\prime}\right) \neq \varnothing$, then $\tilde{v} \subset \tilde{\varphi}_{[-2 \alpha, 2 \alpha]}\left(\tilde{D}^{\prime}\right)$.

(2) $\tilde{D} \times[-3 \alpha, 3 \alpha] \ni(p, t) \mapsto \tilde{\varphi}_{t}(p) \in \tilde{\varphi}_{[-3 \alpha, 3 \alpha]}(\tilde{D})$ is a diffeomorphism. 
LEMma. Let $\tilde{v}, \tilde{v}^{\prime} \in \tilde{V}$. If there are points $\tilde{x} \in \tilde{v}$ and $0<\tau \leqslant \alpha$ with $\tilde{\varphi}_{\tau}(\tilde{x}) \in$ $\operatorname{Int}\left(\tilde{v}^{\prime}\right)$, then there is an oriented path from $\tilde{v}$ to $\tilde{v}^{\prime}$.

Proof. Choose an open neighborhood $U^{\prime}$ of $\tilde{\varphi}_{\tau}(\tilde{x})$ so that $U^{\prime} \cap \tilde{D}^{\prime} \subset \operatorname{Int}\left(\tilde{v}^{\prime}\right)$, and put $U=\tilde{v} \cap \operatorname{Pr}\left(U^{\prime} \cap \tilde{D}^{\prime}\right)$, where $\operatorname{Pr}: \tilde{\varphi}_{[-3 \alpha, 3 \alpha]}(\tilde{D}) \rightarrow \tilde{D}$ is given by $\tilde{\varphi}_{t}(\tilde{z}) \mapsto \tilde{z}$. Since $\Gamma^{\prime}(\tilde{V})$ is dense in $\Gamma(\tilde{V})$, the set $U \cap \Gamma^{\prime}(\tilde{V})$ is not empty. As $\Gamma^{\prime}(\tilde{V})$ is $\tilde{T}$-invariant, we get the conclusion.

Now suppose the distance between $\tilde{v}, \tilde{v}^{\prime} \in \tilde{V}$ is shorter than $8 \alpha C$. Then we can conclude they are joined by a curve in the $\mathrm{CW}$-complex $(\tilde{V}, \tilde{E})$ in the following manner. Put $v=P(\tilde{v})$ and $v^{\prime}=P\left(\tilde{v}^{\prime}\right)$. Since $\varphi_{t}$-periodic points are dense in $\Gamma(V)$, there are $\varphi_{t}$-periodic points $x \in \operatorname{Int}(v)$ and $y \in \operatorname{Int}\left(v^{\prime}\right)$. The point $z=\langle x, y\rangle \in$ $W_{\varepsilon}^{\mathrm{s}}\left(\varphi_{\mu}(x)\right) \cap W_{\varepsilon}^{\mathrm{u}}(y)(|\mu| \leqslant \varepsilon)$ satisfies

$$
d(z, x) \leqslant d\left(z, \varphi_{\mu}(x)\right)+d\left(\varphi_{\mu}(x), x\right) \leqslant 2 \varepsilon C .
$$

Let $\tilde{x} \in \tilde{v}, \quad \tilde{y} \in \tilde{v}^{\prime}, \tilde{z}$ be the points with $\pi(\tilde{x})=x, \pi(\tilde{y})=y, \pi(\tilde{z})=z$, and $d(\tilde{x}, \tilde{z}) \leqslant 2 \varepsilon C$, For some $0 \leqslant \nu \leqslant \alpha, \tilde{\varphi}_{\nu}(\tilde{z})$ is contained in $\Gamma(\tilde{V})$, and is denoted by $\tilde{\Phi}(\xi, 0)$ with some $\xi \in \Sigma(\tilde{V}, \tilde{E})$. Since $x \in \operatorname{Int}(v)$ is a $\varphi_{t}$-periodic point and $\lim _{t \rightarrow \infty} d\left(\tilde{\varphi}_{t}(\tilde{z}), \tilde{\varphi}_{t+\mu}(\tilde{x})\right)=0$, we can find $t_{0}>\alpha, \tilde{v}^{\prime \prime} \in P^{-1}(v)$, and $\omega$ with $|\omega| \leqslant \alpha$ such that

$$
\tilde{\varphi}_{t_{0}+\mu}(\tilde{x}), \tilde{\varphi}_{t_{0}+\omega}(\tilde{z}) \in \operatorname{Int}\left(\tilde{v}^{\prime \prime}\right)
$$

Choose a positive integer $n$ and positive $\tau(\leqslant \alpha)$ so that $\tilde{\varphi}_{\tau}\left\langle\Phi\left(\sigma^{n} \xi, 0\right)\right\rangle=\tilde{\varphi}_{t_{0}+\omega}(\tilde{z})$, where $\sigma$ denotes the shift operator. By the previous lemma, we have an oriented path $\left(\tilde{v}_{0}, \ldots, \tilde{v}_{m}\right)$ from $\xi^{n}$ to $\tilde{v}^{\prime \prime}$. Hence $\xi^{0}$ and $\tilde{v}^{\prime \prime}$ are joined by an oriented path $\left(\xi^{0}, \ldots, \xi^{n}, \tilde{v}_{1}, \ldots, \tilde{v}_{m}\right)$. Similarly there is an oriented path joining $\tilde{v}$ and $\tilde{v}^{\prime \prime}$. Therefore we get a curve (not an oriented path) from $\tilde{v}$ to $\xi^{0}$ in $(\tilde{V}, \tilde{E})$. Applying the same argument to $\tilde{z}$ and $\tilde{y}$, we can conclude that $\tilde{v}$ and $\tilde{v}^{\prime}$ are joined by a curve in $(\tilde{V}, \tilde{E})$.

Given arbitrary $\tilde{v}, \tilde{w} \in \tilde{V}$, we choose points $p_{0}, \ldots, p_{s}$ in $\tilde{M}$ so that $p_{0} \in \tilde{v}$, $p_{s} \in \tilde{w}$, and $d\left(p_{j-1}, p_{j}\right)<5 \alpha C$. There are $\tilde{v}_{j} \in \tilde{V}$ and $0 \leqslant \tau_{j} \leqslant \alpha$ with $\tilde{\varphi}_{\tau_{j}}\left(p_{j}\right) \in \tilde{v}_{j}$, $j=1, \ldots, s-1$. Since the distance between $\tilde{v}_{j-1}$ and $\tilde{v}_{j}$ is smaller than $8 \alpha C, \tilde{v}$ $\left(=\tilde{v}_{0}\right)$ and $\tilde{w}\left(=\tilde{v}_{s}\right)$ are joined by a curve in $(\tilde{V}, \tilde{E})$. Hence we obtain that $(\tilde{V}, \tilde{E})$ is connected.

2. Proof of theorem. We prove the theorem by using the covering of connected graphs $(\tilde{V}, \tilde{E}) \rightarrow(V, E)$. Since $(V, E)$ is irreducible, there exist oriented closed paths $c_{1}, \ldots, c_{m}$ whose homotopy classes generate the fundamental group $\pi_{1}(V, E)$ of the CW-complex (see [2, Lemma 1-1]). Here we recall how $(V, E)$ approximates the flow $\varphi_{t}$. Choose a point $x(v) \in v$ for each $v \in V$, and definé a continuous map $\iota$ : $(V, E) \rightarrow M$ by $\iota(v)=x(v)$ and $\iota(v, w)=($ the minimal geodesic joining $x(v)$ and $x(w))$. By Bowen's result [4] we get that for each oriented closed path $c$ the loop $\iota(c)$ is free homotopic to some $\varphi_{t}$-orbit cycle (see [2] for details). Therefore there are $\varphi_{t}$-closed orbits $\mathfrak{p}_{j}$ such that $\iota\left(c_{j}\right)$ is free homotopic to $\mathfrak{p}_{j}^{k_{j}}$ for some positive integer $k_{j}$. Hence the free homotopy classes of $\mathfrak{p}_{j}$ generate $\iota_{*}\left(\pi_{1}(V, E)\right)$. What we have to do is to check that $\iota_{*}$ is surjective. Let $\tilde{v} \in \tilde{V}$. The connectedness of $(\tilde{V}, \tilde{E})$ 
guarantees that for each $\gamma \in \pi_{1}(M)$ there is a curve $l$ from $\tilde{v}$ to $\gamma \tilde{v}$ for each $\gamma \in \pi_{1}(M)$. Since $\tilde{v}$ is contained in a covering sheet, the homotopy class of $\iota(P(l))$ coincides with $\gamma$. Hence $\iota_{*}$ is surjective and we get the theorem.

3. A remark on the analyticity of $L$-functions. It has been noted that the zeta function of an Anosov flow $\varphi_{t}: M \rightarrow M$ is useful in the study of $\varphi_{t}$-closed orbits (for example see [3, 5, 7, and 10]). As an analogue of Selberg's zeta functions, we [3] defined the $L$-function for a flow $\psi_{i}: X \rightarrow X$ associated to an $N$-dimensional unitary representation $\rho: \pi_{1}(X) \rightarrow U(N)$ by

$$
L\left(s ; \rho, \psi_{t}\right)=\prod_{\mathfrak{p}} \operatorname{det}\{I-\rho(\langle\mathfrak{p}\rangle) \exp \langle-s \tau(\mathfrak{p})\rangle\}^{-1}
$$

where $\mathfrak{p}$ runs over all $\psi_{t}$-closed orbits and $\tau(\mathfrak{p})$ denotes the minimal period of $\mathfrak{p}$.

Let $h\left(\varphi_{t}\right)$ denote the topological entropy of $\varphi_{t}$. Combining Theorem D in [3] with our theorem, we can conclude

Proposition. Let $\varphi_{t}: M \rightarrow M$ be a transitive Anosov flow. If $\rho: \pi(M) \rightarrow U(N)$ is irreducible and $N \geqslant 2$, then the L-function $L\left(s ; \rho, \varphi_{t}\right)$ is holomorphic in some neighborhood of $\mathscr{R} e s \geqslant h\left(\varphi_{t}\right)$.

This result improves Theorem B in [3]. Actually by Manning-Bowen's counting lemma [4], the problem of the analyticity of $L\left(s ; \rho, \varphi_{t}\right)$ was reduced to that of $L\left\langle s ; \rho \circ \iota_{*}, \sigma(f)_{t}\right\rangle$; here $\sigma(f)_{t}$ denotes the suspension flow on $\Sigma(V, E, f)$. By Theorem $\mathrm{D}$ in [3], if $N \geqslant 2$ and $\rho \circ \iota_{*}$ is irreducible then $L\left\langle s ; \rho \circ \iota_{*}, \sigma(f)_{t}\right\rangle$ is holomorphic in some neighborhood of $\mathscr{R} e s \geqslant h\left\langle\sigma(f)_{t}\right\rangle=h\left(\varphi_{t}\right)$. Since $\iota_{*}: \pi_{1}(V, E)$ $\rightarrow \pi_{1}(M)$ is surjective, $\rho \circ \iota_{*}$ is irreducible if and only if $\rho$ is irreducible, hence we get the assertion.

\section{REFERENCES}

1. T. Adachi, Markov families for Anosov flows with an involutive action, Nagoya Math. J. 104 (1986), $55-62$.

2. T. Adachi and T. Sunada, Homology of closed geodesics in a negatively curved manifold, J. Differential Geom. (to appear).

3. Twisted Perron-Frobenius theorem and L-functions, J. Funct. Anal. (to appear).

4. R. Bowen, Symbolic dynamics for hyperbolic flows, Amer. J. Math. 95 (1973), 429-460.

5. D. Fried, Flow equivalence, hyperbolic systems and new zeta function for flows, Comment. Math. Helv. 57 (1982), 237-259.

6. The zeta functions of Ruelle and selberg. I (preprint).

7. W. Parry and M. Pollicott, The Chebotarev theorem for Galois coverings of Axiom A flow, Ergodic Theory Dynamical Systems 6 (1986), 133-148.

8. C. Pugh and M. Shub, $\Omega$-stability theorem for flows, Invent. Math. 11 (1970), 150-158.

9. S. Smale, Differential dynamical systems, Bull. Amer. Math. Soc. 73 (1967), 747-817.

10. T. Sunada, Geodesic flows and geodesic random walks, Geometry of Geodesics and Related Topics, Studies in Pure Math., no. 3, North-Holland, Amsterdam and New York, 1984, pp. 47-86.

Department of Mathematics, Nagoya University, Nagoya 464, JaPan

Current address: Department of Mathematics, Faculty of General Education, Kumamoto University, Kumamoto 860, Japan 